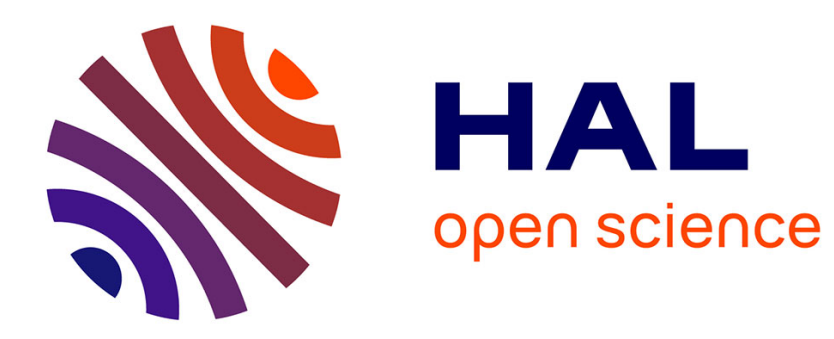

\title{
Programmation réactive en OCaml
}

Christophe Deleuze

\section{To cite this version:}

Christophe Deleuze. Programmation réactive en OCaml. Journal Européen des Systèmes Automatisés

(JESA), 2009, 43 (7-8-9), pp.757-771. hal-00493223

\section{HAL Id: hal-00493223 \\ https://hal.science/hal-00493223}

Submitted on 18 Jun 2010

HAL is a multi-disciplinary open access archive for the deposit and dissemination of scientific research documents, whether they are published or not. The documents may come from teaching and research institutions in France or abroad, or from public or private research centers.
L'archive ouverte pluridisciplinaire HAL, est destinée au dépôt et à la diffusion de documents scientifiques de niveau recherche, publiés ou non, émanant des établissements d'enseignement et de recherche français ou étrangers, des laboratoires publics ou privés. 


\title{
Programmation réactive en OCaml
}

\author{
Christophe Deleuze
}

Laboratoire de conception et d'intégration des systèmes (LCIS)

50 , rue Barthélémy de Laffemas, BP54

F-26902 Valence Cedex 09

christophe.deleuze@lcis.grenoble-inp.fr

RÉSUMÉ. La programmation réactive permet d'écrire des programmes sous forme d'un ensemble de processus qui s'exécutent de manière synchronisée et communiquent par diffusion de signaux. Ce paradigme peut être fourni par des langages spécialisés (parfois basés sur des langages "classiques") ou par des bibliothèques. Le langage ReactiveML est un tel langage réactif basé sur OCaml. Nous décrivons ici une bibliothèque OCaml fournissant les constructions réactives de ReactiveML pour le langage OCaml lui-même. Les processus devront pour cela être rédigés en style trampoline. Des exemples montrent que le style obtenu est raisonnable et que les performances sont au moins équivalentes.

ABSTRACT. Reactive programming allows to write programs as a set of processes executing synchronously and communicating by signal broadcasting. This paradigm can be provided by specialized languages (sometimes based on "classic" languages) or by a library. ReactiveML is such a language based on OCaml. We describe an OCaml library that provides ReactiveML reactive constructs for the OCaml language. Processes must be written in trampolined style. Examples show the resulting style to be acceptable, and performances at least as good.

MOTS-CLÉS : programmation réactive, ReactiveML, langage fonctionnel, OCaml, style trampoline, bibliothèque.

KEYWORDS: reactive programming, ReactiveML, functionnal language, OCaml, trampolined style, library.

JESA - 43/2009. MSR 2009, pages 757 à 771 


\section{Introduction}

\section{Programmation réactive}

Les langages synchrones ont été introduits dans les années 80 pour programmer des systèmes réactifs : systèmes qui interagissent continuellement et en temps réel avec leur environnement (Halbwachs, 1993). En particulier, Estérel (Boussinot et al., 1991) adopte le paradigme impératif. Il permet d'exprimer un programme comme un ensemble de processus concurrents, synchronisés sur une notion d'instant. À chaque instant chacun des processus a l'opportunité de s'éxécuter. La communication entre les processus se fait par diffusion de signaux valués. À chaque instant, un signal est soit présent (et porteur d'une ou plusieurs valeurs) soit absent, et tous les processus en ont la même vision.

Une forte limitation du modèle synchrone est l'impossibilité de créer dynamiquement des processus ou de réaliser des calculs complexes impliquant des boucles ou l'usage de la récursion : c'est à ce prix que l'aspect temps réel du système peut être garanti. Beaucoup d'applications pourraient bénéficier du modèle réactif sans pour autant demander des contraintes temporelles fortes comme les systèmes réactifs synchrones. À partir de cette idée Frédéric Boussinot a introduit le modèle réactif (sous entendu non synchrone) qui reprend les mêmes notions en ajoutant essentiellement la possibilité de créer des processus, appelée composition dynamique (Boussinot, 1996). Ces idées ont tout d'abord été mises en œuvre dans le langage ReactiveC (Boussinot, 1991) puis en Java (Boussinot et al., 2000) et ont plus récemment abouti à la proposition des FairThreads (Boussinot, 2006).

OCaml (Leroy, 2008) est un langage fonctionnel (avec des traits impératifs et orientés objet) de la famille ML. Nous supposons le lecteur raisonnablement familier avec ce langage. ReactiveML (Mandel, 2006) est une extension de OCaml ajoutant des constructions réactives inspirées d'Estérel. Le compilateur ReactiveML génère du code OCaml pour différents runtimes qui sont essentiellement des machines réactives écrites en OCaml.

\section{Trampoline}

Dans un programme une continuation représente "ce qui reste à exécuter" à un point donné (Reynolds, 1993). Le style CPS (continuation passing style) (Steele et $a l ., 1976$ ) consiste à écrire (ou transformer) un programme de façon à rendre explicite toutes les continuations. Une fonction ne retourne jamais, elle transmet son résultat à sa continuation, qui est une fonction qu'elle a reçue en paramètre. L'exécution ne requiert donc pas de maintenir une pile des appels de fonctions, chaque appel de fonction écrasant l'enregistrement d'activation précédent.

Le style trampoline (Ganz et al., 1999) se base sur le CPS pour mettre en œuvre un ordonnancement coopératif entre plusieurs processus. Ceci est illustré par la figure 1 qui montre un exemple de calcul de la factorielle en style trampoline. L'appel de bounce constitue un point de coopération et son argument est la continuation du 
processus, qui sera utilisée par l'ordonnanceur (appelé ici seesaw) pour le réactiver. Dans cet exemple, la factorielle est exécutée concurremment avec un autre processus.

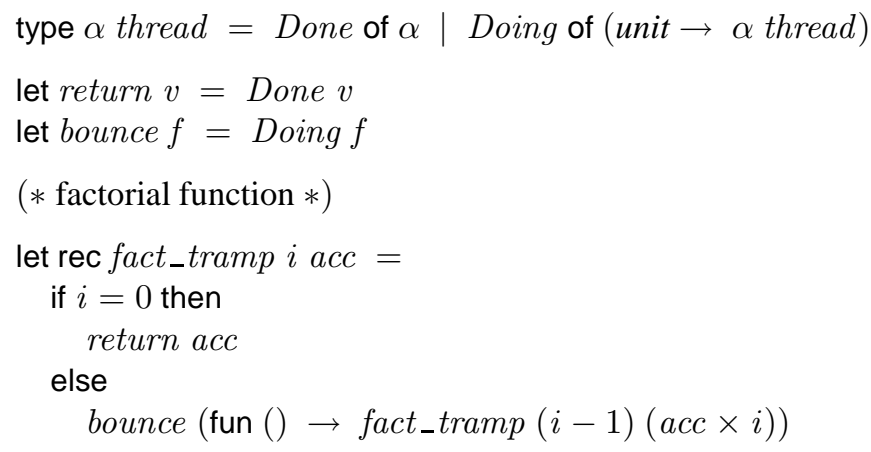

( $*$ run two computations concurrently. Return result of whichever terminates first, and rest of other computation $*$ )

let rec seesaw $f 1$ f2 $=$ match $f 1$ () with

Done $v \rightarrow v$, f2

Doing $f \rightarrow$ seesaw f2 $f$

let rec dotter ()$=$ print_char '.'; bounce dotter

( $*$ compute factorial, printing dots at each step $*$ )

let fact $n=$

seesaw dotter (fun ()$\rightarrow$ fact_tramp $n 1$ )

Figure 1. Style trampoline

On voit donc qu'il est possible (et facile) d'exécuter des processus concurrents à partir du moment où chacun d'eux est écrit en style trampoline.

Programmation réactive en OCaml

Nous avons développé une bibliothèque permettant la programmation réactive en OCaml. Celle-ci met en œuvre l'ordonnancement et les communications entre processus, en reprenant le modèle de ReactiveML. Les processus devront être écrits en style trampoline, et feront appels aux fonctions de la bibliothèque.

Fournir ces mécanismes au sein du langage plutôt que par une extension apporte un certain nombre d'avantages. L'intégralité du langage est disponible (ReactiveML ne supporte pas les foncteurs ni les objets), ainsi que les outils associés (débogueur, profileur, environnement de développement). D'autre part la bibliothèque peut être utilisée avec des extensions de OCaml comme MetaOCaml (Taha, 2004). D'un autre côté, au contraire d'une bibliothèque, un langage spécifique comme ReactiveML peut 
adapter la syntaxe aux nouveaux concepts introduits ${ }^{1}$ et surtout réaliser à la compilation des vérifications sémantiques spécifiques.

Dans la section suivante, nous présentons les principales constructions de ReactiveML et leurs équivalents pour notre bibliothèque. La section 3 décrit deux exemples de programmes réactifs écrits à l'origine en ReactiveML. La section 4 décrit les grandes lignes de l'implémentation de la bibliothèque. La section 5 présente une évaluation des performances réalisée sur les deux exemples. Conclusion et perspectives terminent l'article.

\section{Constructions réactives en style trampoline}

ReactiveML fournit un ensemble de constructions syntaxiques pour les processus. La bibliothèque les met en œuvre sous forme de fonctions. Pour pouvoir être manipulé, un processus sera représenté le plus souvent par une fonction "glaçon" (thunk). L'instruction d'exécution run sera donc réalisée simplement par la fonction let run $p=p()$. Nous noterons RML les fragments de code ReactiveML et TML (pour trampoline ML) ceux pour la bibliothèque.

\section{Trampoline}

Le style trampoline consiste à rendre explicite les continuations aux endroits où le processus est susceptible de se suspendre. Par exemple l'instruction pause indique que le processus attend l'instant suivant pour continuer son exécution. Un autre est l'attente (await) de l'émission d'un signal $s$ : le processus sera relancé au prochain instant suivant l'émission du signal. La valeur du signal est reçue en paramètre par la fonction de continuation. Le processus demo ci-dessous (à gauche RML, à droite TML) affiche un message au premier instant puis attend l'émission du signal $s 1$ et affiche la valeur émise. term permet de terminer le processus.

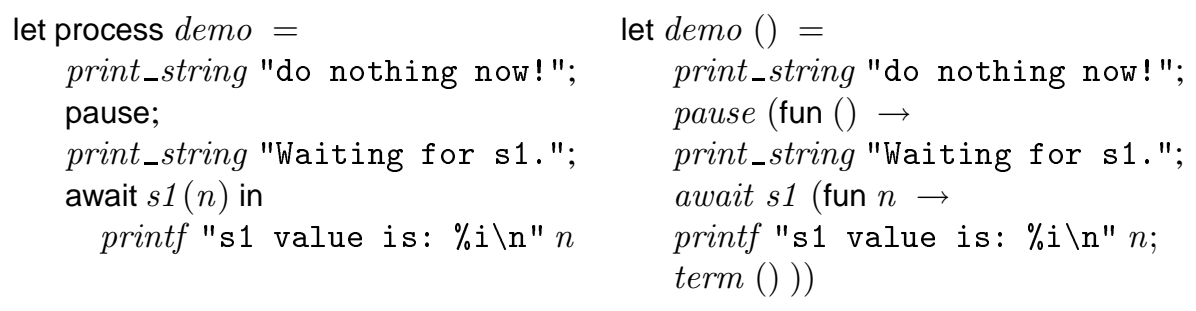

Certaines constructions nécessitent de "factoriser" le code d'une continuation "lointaine" commune. C'est le cas de l'instruction present qui exécute instantanément (c'est-à-dire dans l'instant courant) la partie c_then si le signal est présent dans l'instant, la partie c_else à l'instant suivant dans le cas contraire (ce retard de réaction à l'absence d'un signal permet d'éviter les problèmes de causalité).

1. Quoique OCaml dispose des outils Camlp4 et Camlp5 pour étendre sa syntaxe. 
Constructions de contrôle

Trois constructions de contrôle agissent sur l'exécution des processus. Elles sont réalisées en TML sous forme d'une fonction. Par exemple, do/until est la construction de préemption : le processus contenu est préempté (terminé) en fin d'instant si le signal associé a été émis. Les deux autres constructions (suspension et désactivation) sont traitées de la même façon.

do

print_string "Wait for s..."; await $s$;

print_string "Arrived!"

until $s^{\prime}$

print_string "Left do/until!"; $\cdots$ dountil $s^{\prime}$

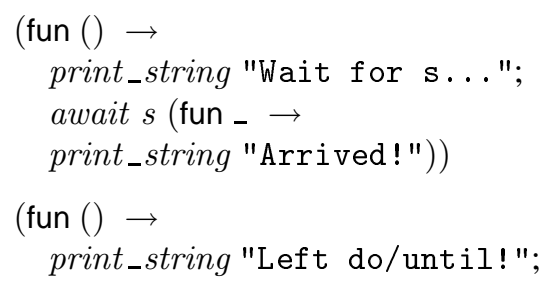

\section{Exemples}

Sieve

Le crible d'Ératosthène est un exemple classique de l'approche réactive d'abord proposé dans le contexte d'un langage flot de données (Kahn et al., 1977). Le crible se construit comme une chaîne de processus filter encadrés par un générateur (integers) d'un côté, un récepteur (output) précédé d'un "étendeur" (shift) de l'autre, comme illustré figure 2 qui montre l'état initial et après la création du filtre sur 2. Les nombres progressent d'une étape de la chaîne à chaque instant.
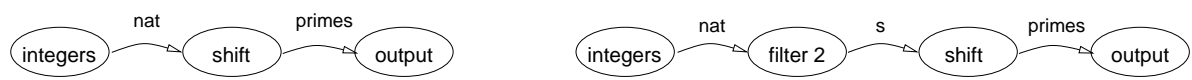

Figure 2. Les processus du crible

Détaillons chacun des processus (figure 3) :

- integers génère sur un signal la suite des entiers à partir de 2.

- filter filtre les multiples de son paramètre : il reçoit des entiers sur son signal d'entrée et réemet ceux qu'il ne filtre pas sur son signal de sortie. La boucle infinie de RML est ici exprimée par la récursion.

- shift reçoit des entiers sur son signal d'entrée, crée un nouveau signal (appelé $s$ localement) et insère un processus filter dans la chaîne à chaque nouveau nombre reçu (qui sera premier par construction de la chaîne). On utilise ici la fonction tail_par.

- output, placé en fin de chaîne, est chargé de l'affichage du nombre premier qui a passé tous les filtres. Il termine le programme quand le nombre reçu atteint une valeur limite. L'appel à Mem. exit affiche la mémoire allouée dans le tas avant de terminer. 


\section{sieve.rml}

let llast $=$ ref 0

let show $=$ ref true

let rec process integers $n s_{-}$out $=$ emit $s_{\text {_out }} n$;

pause;

run (integers $(n+1) s_{-}$out)

let process filter prime $s_{-}$in $s_{-}$out $=$ loop

await one $s_{-} i n(n)$ in

if ( $n$ mod prime) $\not \equiv 0$ then

emit $s_{\text {_out }} n$

end

let rec process shift $s_{-}$in $s_{-}$out $=$ await one $s_{-}$in (prime) in

emit $s_{-}$out prime;

signal $s$ in

run (filter prime $s_{-}$in $s$ )

||

run (shift $s s_{-}$out)

let process output $s_{-} i n=$ loop

await one $s_{-}$in $(n)$ in

if $n \geq$ !llast then Mem.exit ();

if ! show then begin

print_int $n$;

print_string " ";

flush stdout

end

end

let process sieve $=$

Arg.parse [...]

(fun _ $\rightarrow($ )) "sieve";

signal nat in

signal primes in

run (integers 2 nat)

|l

run (shift nat primes)

|l

run (output primes)

\section{sieve.ml}

module $T M L=$

Tml.Gen_TML(struct type $t=i n t$ end $)$

open $T M L$

let last $=$ ref 0

let show = ref true

let rec integers $n s_{-}$out ()$=$

emit s_out $n$;

pause (integers $(n+1) s_{-}$out)

let rec filter prime $s_{-}$in $s_{-}$out ()$=$ await_one $s_{-}$in (fun $n \rightarrow$ if $(n$ mod prime $) \not \equiv 0$ then emit s_out $n$; run (filter prime $s_{-}$in $s_{-}$out))

let rec shift $s_{-}$in $s_{-}$out ()$=$ await_one $s_{-}$in (fun prime $\rightarrow$ emit s_out prime;

let $s=$ signal () in

tail_par (filter prime $s_{-}$in $s$ )

(shift $s s_{-}$out))

let rec output $s_{-}$in ()$=$ await_one $s_{-}$in (fun $n \rightarrow$

if $n \geq$ !last then Mem.exit ();

if ! show then begin

$$
\begin{aligned}
& \text { print_int n; } \\
& \text { print_string " "; } \\
& \text { flush stdout }
\end{aligned}
$$

end;

run (output $s_{-}$in))

let sieve ()$=$

let $n a t=$ signal () in

let primes $=$ signal () in

tail_parn [integers 2 nat;

shift nat primes; output primes ]

Arg.parse [... ]

(fun _ $\rightarrow())$ "sieve";

start [ sieve ]

Figure 3. Code pour le crible, en RML et TML 
- sieve, processus initial qui amorce le système. On remarque l'utilisation du tail_parn, similaire à tail_par mais prenant une liste de processus en paramètre. start démarre les processus passés en paramètre (en RML le processus initial est fixé à la compilation).

Tous les signaux portant des entiers, le type passé au foncteur est simplement int. On note que la formulation des processus comme glaçons amène souvent une notation élégante et proche de celle de ReactiveML.

Elip

Elip est un logiciel de simulation de protocole de routage pour réseaux ad'hoc, écrit en ReactiveML (Mandel et al., 2005). Chaque nœud du réseau est implémenté par un processus. Il a pu très facilement être porté sur TML. Sur les 4300 lignes de code, seules 270 concernent les définitions de processus (au nombre de 11) et doivent donc être converties en style trampoline. Aucune difficulté notable n'a été rencontrée.

Le programme utilise 10 signaux, dont 4 non valués. Les autres portent des articles (types position et node), une paire de flottants, un caractère ou un entier. Le module paramètre du foncteur sera donc le suivant :

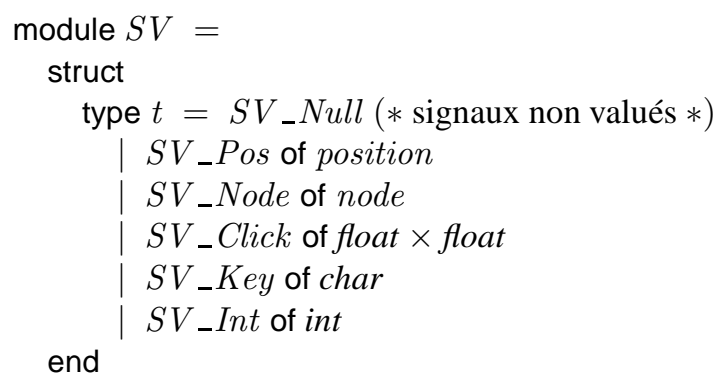

L'émission d'un signal devra utiliser le constructeur correspondant, par exemple emit click $\left(S V_{-} C l i c k\left(p o s_{\_} x, p o s_{-} y\right)\right)$.

\section{Réalisation de la bibliothèque}

Nous décrivons ici les grandes lignes d'une version simplifiée de la bibliothèque. Le code détaillé est disponible sous forme d'un document "litterate programming" (Deleuze, 2009) montrant la mise en œuvre progressive des fonctionnalités. La bibliothèque complète comprend environ 500 lignes de code.

Principes

Les processus sont des fonctions glaçons retournant des valeurs de type coop indiquant la raison de la suspension et la continuation éventuelle. 


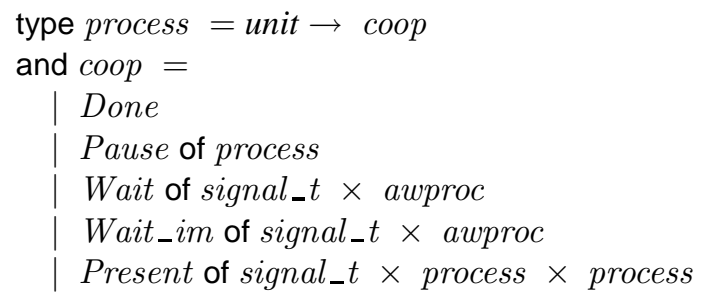

awproc (pour awaiting process) est le type des processus attendant l'arrivée d'un signal (sans se préoccuper des valeurs éventuelles portées), l'intégralité des valeurs émises sur le signal ou une seule des valeurs émises. sval_t est le type somme des valeurs de signaux, paramètre du foncteur.

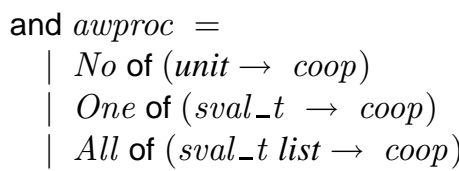

Les fonctions de suspension que nous avons vues précédemment consistent essentiellement à retourner une valeur de type $\operatorname{coop}$ (ispst indique si un signal a déjà été émis dans l'instant courant).

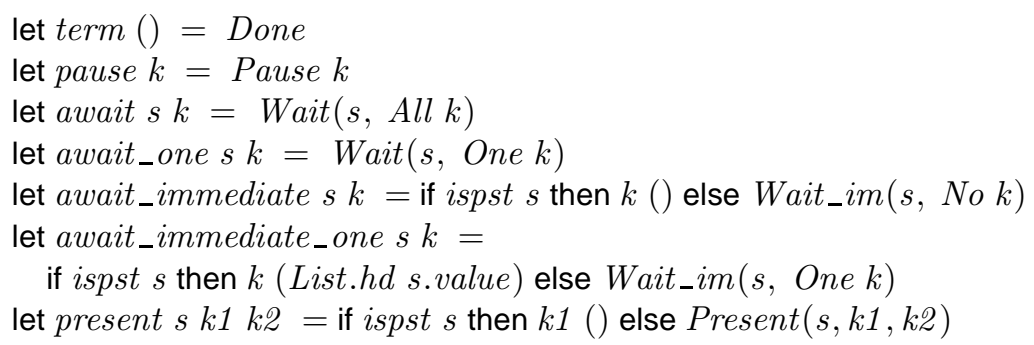

Les processus sont stockés dans diverses listes :

- runq: processus en attente d'exécution sur l'instant,

- pauseq : processus en attente de l'instant suivant,

- à chaque signal sont associées trois listes present, await_im et await contenant les processus en attente sur ce signal.

Au cours de chaque instant l'ordonnanceur (sched) lance un à un les processus de la liste runq et récupère les continuations (dans des valeurs de type coop) qu'il place dans la liste appropriée (pauseq si le processus a terminé l'instant, une liste d'attente associée à un signal si le processus attend ce signal, voir figure 4a). Si un processus émet un signal, les processus en attente immédiate (await_im et present) sur ce signal sont immédiatement replacés dans runq.

L'instant se termine quand l'ordonnanceur trouve runq vide. Il exécute alors la fonction next_instant qui transfère le contenu de pauseq dans runq ainsi que les 
processus qui ne doivent plus être en attente à l'instant suivant (await sur un signal présent, present sur un signal absent, figure $4 \mathrm{~b}$ ).

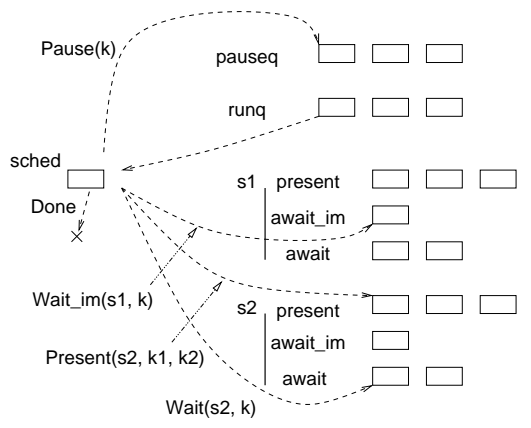

a) en cours d'instant

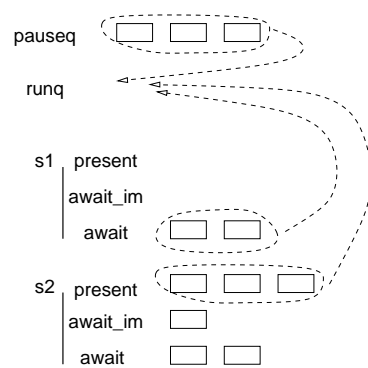

b) en fin d'instant

Figure 4. Utilisation des listes de processus

Création de processus

Les fonctions de création de processus ( $p a r$ et ses variantes) se basent sur la fonction spawn qui ajoute un processus dans runq et sur par_help qui met en place la synchronisation des deux processus. parn et tail_parn sont réalisées de façon similaire.
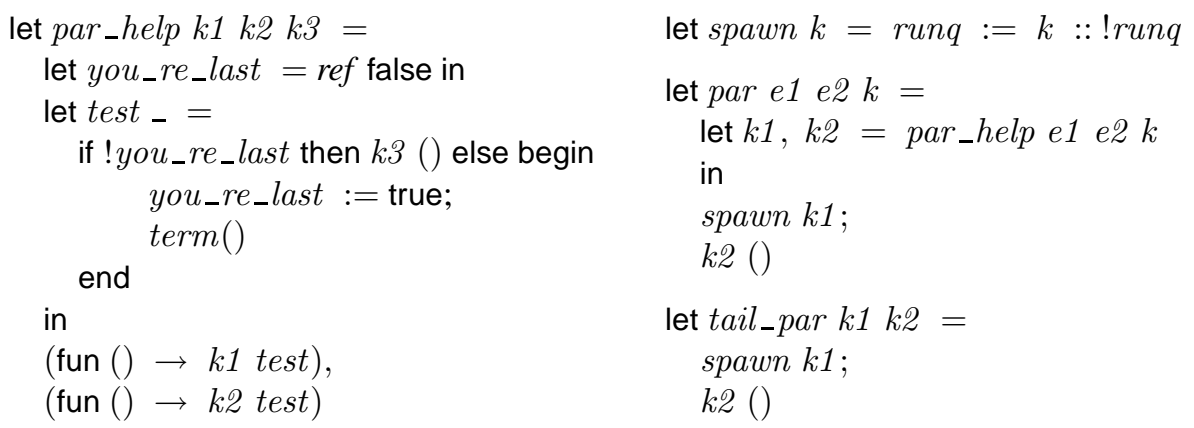

\section{Signaux}

Un signal est une structure contenant des champs mutables dont les deux derniers instants où le signal a été émis (permet de tester le status du signal à l'instant courant et à l'instant précédent), les deux dernières (listes de) valeurs émises (de type sval_t, le type fourni en paramètre au foncteur), une fonction de collecte des valeurs émises au cours d'un instant et les trois listes de processus en attente.

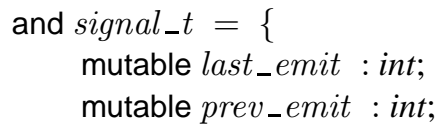




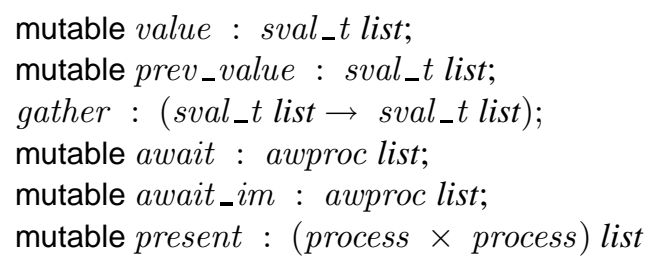

Pour limiter les traitements en fin d'instant la fonction next_instant ne considère que les signaux présents dans la liste used_signals. Ces signaux utilisés sont ceux qui ont été émis sur cet instant ou pour lesquels un processus est en attente dans la liste present. Cette liste est remise à vide pour l'instant suivant.

Constructions de contrôle

Chaque processus est associé à une pile de contexte indiquant les conditions dans lesquelles il peut être suspendu, préempté ou désactivé ainsi que la continuation à lancer le cas échéant.

and contextelement $=$

Until of signal_t $\times$ process

Control of signal_t $\times$ process

When of signal_t $\times$ awproc

and ctxt $=$ contextelement list

L'implémentation réelle est un peu plus complexe, le nombre de contextes de préemption ou de désactivation étant maintenu avec la liste des contextes pour être accessible sans parcours de la liste.

La présence des contextes complique la gestion des signaux. En effet, un processus soumis à un do/until qui doit être préempté en fin d'instant peut se trouver dans n'importe quelle liste. La notion de signaux utilisés est étendue et de nouvelles listes sont mises en place pour limiter le plus possible le coût des opérations de fin d'instant. Nous renvoyons le lecteur à (Deleuze, 2009) pour les détails.

À titre d'exemple, la fonction dountil $s$ e $k$ a pour effet de placer en tête de runq un processus $e$ dans un contexte préempté par $s$ et avec la continuation $k$, puis de terminer (avec une nouvelle valeur de coopération Next). L'ordonnanceur va relancer depuis runq le processus dans le nouveau contexte. Quand $e$ se termine, le sommet de sa pile de contexte est dépilé et sa continuation lancée.

let dountil $s$ e $k=$

let cel $=$ !current_context in

put_in_runq $(($ cel @ $[\operatorname{Until}(s, k)]), e) ; N e x t$

Les processus suspendus sont placés dans la liste await_im du signal correspondant. En fin d'instant la pile de contexte de chaque processus est examinée et les 
opérations nécessaires sont effectuées (préemption, désactivation ou réactivation éventuellement sur plusieurs niveaux).

\section{Performances}

Nous comparons les performances, en OCaml code-octet et natif, des trois runtimes de RML (Lco_ct, Lco_ct_class et Lk) avec trois versions de notre bibliothèque TML :

- TML4 version sans constructions de contrôle

- TML5 constructions de contrôle non imbriquées

- TML6 constructions de contrôle imbriquées.

Les mesures décrites ci-dessous ont été effectuées sur des machines équipées d'un processeur Intel Core 2 Duo à 2,33 Ghz et 2 Go de mémoire, utilisant un noyau linux 2.6.24, OCaml 3.09.2, RML 1.07.1. Le temps de calcul a été mesuré par la commande time et l'occupation mémoire a été fournie par le module $G c$ de OCaml.

Sieve

Les figures 5 et 6 montrent les performances pour l'application sieve. Le temps d'exécution est avantageux pour TML avec les versions incomplètes de la bibliothèque, particulièrement en cas de la compilation native. Les constructions de contrôle induisent clairement un coût important. En code-octet la situation est moins claire, en particulier TML6 est plus lent que tous les runtimes RML. Il est possible que la gestion (assez complexe) des constructions de contrôle imbriquées par TML ne soit pas la plus efficace possible.
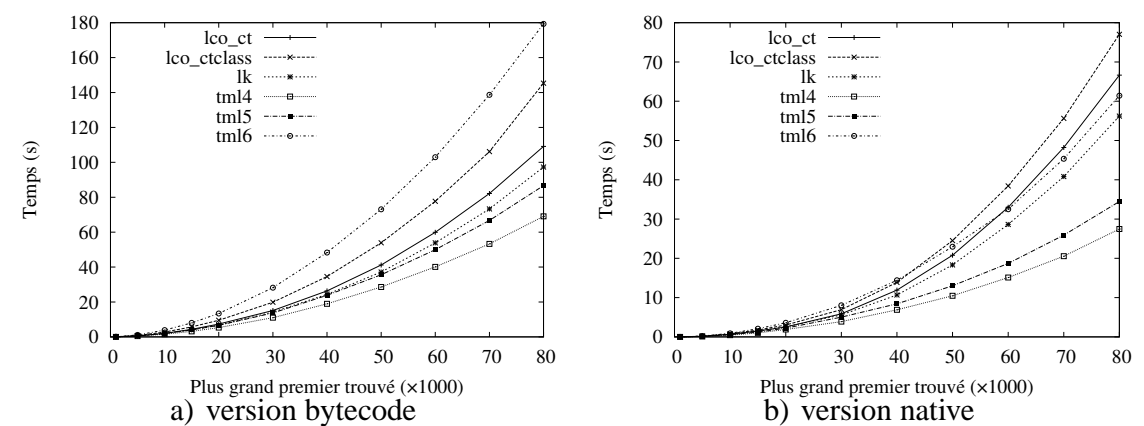

Figure 5. Crible, temps d'exécution

L'occupation mémoire est par contre nettement à l'avantage de TML. Que cela soit en code-octet ou en natif, on observe un gain d'au moins $50 \%$. Les figures ne le montrent pas mais le gain est un peu plus faible si l'on utilise la fonction par au lieu de 
tail_par (dont RML n'a pas d'équivalent) mais reste conséquent (24\% en code-octet et $43 \%$ en natif).

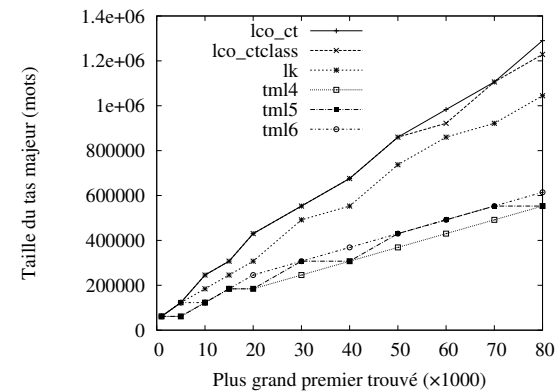

a) version bytecode

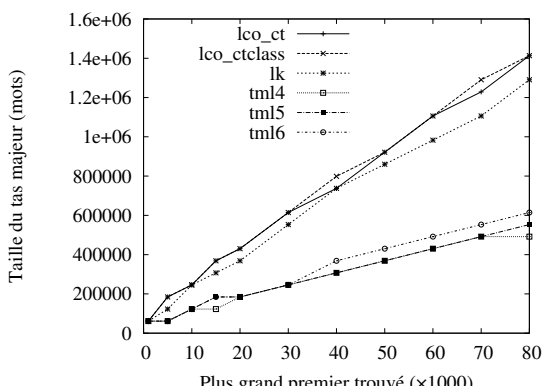

b) version native

Figure 6. Crible, espace mémoire

Elip

elip contient des instructions de contrôle imbriquées, seul TML6 est alors utilisable (figure 7). Globalement, la différence entre RML et TML est ici beaucoup moins nette. En ce qui concerne l'occupation mémoire, nous ne montrons pas les graphes par manque de place, les implémentations sont extrêmement proches. Nous supposons qu'ici l'avantage de TML est noyé dans la masse de mémoire allouée par l'application elle même, alors que dans le cas de sieve l'application elle même n'allouait que très peu de mémoire, la différence entre les implémentations apparaissait alors très nettement. Cette interprétation est confirmée par le fait que tous les runtimes RML ont le même comportement alors que des différences étaient perceptibles avec le crible. Par contre on peut relever un léger avantage en temps pour TML dans le cas de compilation native.

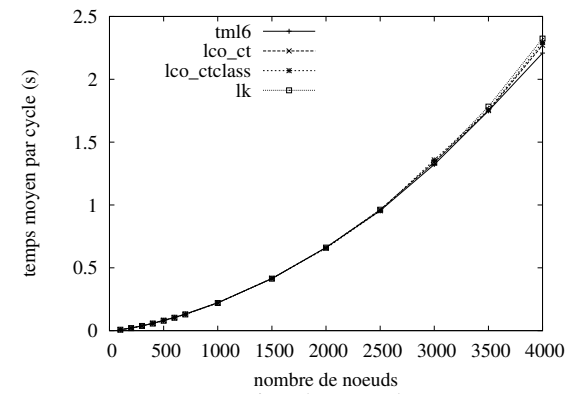

a) version bytecode

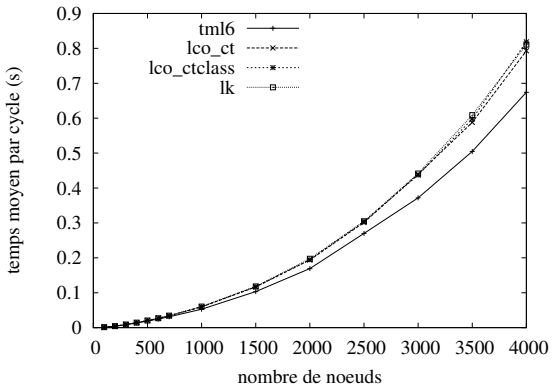

b) version native

Figure 7. Elip, temps d'exécution 
Les simulations ont été exécutées sur 500 cycles avec une densité constante de 20, une graine du générateur aléatoire égale à 0 , et sans l'interface graphique $(-\mathrm{n}$ nodes -N 500 -D 20 -seed 0 -nox).

\section{Conclusion et perspectives}

Le but que nous nous étions fixé était de reproduire les fonctionnalités de ReactiveML en OCaml pur et de voir si le style et les performances étaient raisonnables.

Les exemples que nous avons présentés nous conduisent à penser que le style trampoline est raisonnablement utilisable avec un peu de pratique. Notons que l'on peut aussi adopter un style monadique en définissant simplement un opérateur infixe "bind" par let $(>>=)$ inst $k=$ inst $k$. On obtient une formulation très similaire aux systèmes de threads basés sur les monades comme Lwt (Vouillon, 2008). Le processus integers du crible, par exemple, pourra alors s'écrire :

let rec integers $n s_{-}$out ()$=$

emit s_out $n$;

pause $>>$ integers $(n+1) s_{-}$out

Le style trampoline consiste à faire apparaître explicitement les continuations aux endroits où elles sont nécessaires. Une autre approche consiste à utiliser les continuations implicites en réalisant des captures de continuation. De cette façon la formulation des opérations bloquantes peut se faire en style direct (de la forme let $v=$ await $s$ in ...) et les boucles n'ont pas besoin d'être transformées en fonctions récursives. La bibliothèque caml-shift (Sabry et al., 2008) implémente la capture de continuations partielles ${ }^{3}$ mais elle n'est disponible qu'en code octet et les performances sont sensiblement moins bonnes : sur le crible, nous avons observé un triplement de l'occupation mémoire et du temps de calcul.

Les tests ont montré que les performances sont au minimum honorables, et parfois avantageuses en mémoire. Cette bibliothèque pourrait être préférable à ReactiveML pour des applications utilisant un très grand nombre de processus très simples.

De nombreux travaux s'intéressent à la gestion de la concurrence sans support du système, ou plus généralement à l'ordonnancement coopératif. En particulier FairThreads (Boussinot, 2006) propose un modèle de programmation concurrente inspiré du modèle réactif, et reposant sur des passages de jetons entre threads système. Une implémentation trampoline pourrait être très avantageuse en terme de ressources, notamment pour des applications composées d'un très grand nombre de processus fortement couplés.

Les extraits de code apparaissant dans ce document ont été formatés avec l'outil ocamlweb (modifié pour traiter ReactiveML).

3. Aussi appelées continuations composables ou continuations délimitées. 


\section{Bibliographie}

Boussinot F., « Reactive C: An extension of C to program reactive systems », Software: Practice and Experience, vol. 21, $\mathrm{n}^{\circ}$ 4, p. 401-428, avril, 1991.

Boussinot F., La programmation réactive. Application aux systèmes communicants, Masson et CNET-ENST, 1996.

Boussinot F., « FairThreads: mixing cooperative and preemptive threads in $\mathrm{C}$ », Concurrency and Computation: Practice and Experience, vol. 18, $\mathrm{n}^{\circ}$ 5, p. 445-469, avril, 2006. Also available as a research report.

Boussinot F., de Simone R., « The ESTEREL language », Proceedings of the IEEE, vol. 79, n 9 , p. 1293-1304, septembre, 1991.

Boussinot F., Susini J.-F., « Java threads and SugarCubes », Software: Practice and Experience, vol. $30, \mathrm{n}^{\circ}$ 5, p. 545-566, avril, 2000.

Deleuze C., Programmation réactive en OCaml - Implémentation de la bibliothèque TML, Technical report, LCIS, 2009.

Ganz S. E., Friedman D. P., Wand M., « Trampolined Style», International Conference on Functional Programming, p. 18-27, 1999.

Halbwachs N., Synchronous Programming of Reactive Systems, Kluwer Academic Publishers, 1993.

Kahn G., MacQueen D. B., « Coroutines and networks of parallel processes », Information processing, Toronto, p. 993-998, août, 1977.

Leroy X., The Objective Caml system - Documentation and user's manual, release 3.11, INRIA. 2008.

Mandel L., Conception, Sémantique et Implantation de ReactiveML : un langage à la ML pour la programmation réactive, $\mathrm{PhD}$ thesis, Université Paris 6, mai, 2006.

Mandel L., Benbadis F., « Simulation of Mobile Ad hoc Network Protocols in ReactiveML », Proceedings of Synchronous Languages, Applications, and Programming (SLAP'05), Edinburgh, Scotland, avril, 2005.

Reynolds J. C., « The Discoveries of Continuations », LISP and Symbolic Computation, vol. 6, $n^{\circ} 3-4$, p. 233-247, 1993.

Sabry A., chieh Shan C., Kiselyov O., « Native delimited continuations in (byte-code) OCaml», OCaml library, 2008.

Steele G. L., Sussman G. J., Lambda: The Ultimate Imperative, Technical Report n AIP-353, Massachusetts Institute of Technology, Cambridge, MA, USA, 1976.

Taha W., « A Gentle Introduction to Multi-stage Programming », Domain-Specific Program Generation, Springer Berlin / Heidelberg, p. 30-50, 2004.

Vouillon J., « Lwt: a cooperative thread library », ML '08: Proceedings of the 2008 ACM SIGPLAN workshop on ML, ACM, New York, NY, USA, p. 3-12, 2008. 